\title{
Scintillation of Tantalate Compounds
}

\author{
Edith D. Bourret ${ }^{\mathrm{a}}$, David M. Smiadak ${ }^{\mathrm{a}, \mathrm{b}, *}$, Ramesh B. Borade $^{\mathrm{a}}$, Yunfeng Ma ${ }^{\mathrm{a}}$, Gregory Bizarri ${ }^{\mathrm{a}}$, Marv J. Weber ${ }^{\mathrm{a}}$, \\ Stephen E. Derenzo ${ }^{\mathrm{a}}$ \\ ${ }^{a}$ Lawrence Berkeley National Laboratory, University of California, 1 Cyclotron Rd, Mail stop 55-107, Berkeley, CA, 94720, USA \\ ${ }^{b}$ Department of Chemical Engineering and Material Science, Michigan State University, 2100 Engineering Building, 428 South Shaw Lane, East \\ Lansing, MI, 48824, USA
}

\begin{abstract}
A study of 63 metal-tantalate-oxides was conducted in search of heavy scintillator materials operating at ambient temperature. Tantalates are known to have slow scintillation decay times, however, the high atomic number of tantalum (73) provides good stopping power for gamma rays. The samples were synthesized by solid state reactions. The physical, optical, and scintillation properties of these materials were evaluated by X-ray diffraction, X-ray excited luminescence and pulsed X-ray luminescence. Out of the 63 synthesized tantalates examined, only 12 had luminosity values greater than $1,000 \mathrm{ph} / \mathrm{MeV}$ at room temperature. From these, three compounds $\mathrm{ScTaO}_{4}, \mathrm{YTa}_{3} \mathrm{O}_{9}$, and $\mathrm{Zn}_{3} \mathrm{Ta}_{2} \mathrm{O}_{8}$ have greater than $40 \%$ of their emission in the first $\mu$ s. The brightest and fastest compound of those tested was $\mathrm{Zn}_{3} \mathrm{Ta}_{2} \mathrm{O}_{8}$ with an estimated luminosity of $26,000 \mathrm{ph} / \mathrm{MeV}$ and a main decay time of $600 \mathrm{~ns}$ from its crystalline powder. Further attention is given to $\mathrm{Zn}_{3} \mathrm{Ta}_{2} \mathrm{O}_{8}$ and $\mathrm{Mg}_{4} \mathrm{Ta}_{2} \mathrm{O}_{9}$ scintillation properties, demonstrating their potential for scintillation applications.
\end{abstract}

Keywords: Zinc, magnesium, tantalate, decay time, luminescence, scintillator.

\section{Introduction}

High energy physics has been a driving force for the development and optimization of heavy and fast scintillation materials due to the challenging requirements of modern experiments and the large volume of scintillators required $[1,2] . \mathrm{PbWO}_{4}$, with its combination of high density and fast luminescence decay time makes it one of the most utilized scintillators in current high energy physics. Its luminosity is low (200 ph/MeV) but decays rapidly (6 ns). Among the tungstates, $\mathrm{ZnWO}_{4}$ is relatively bright $(21,500 \mathrm{ph} / \mathrm{MeV})$ but the luminescence decay is slow $(22 \mu \mathrm{s})$ [2]. Additionally, heavy scintillators that provide good stopping power are now in demand for high energy radiography. The brighter $\mathrm{CdWO}_{4}$ and $\mathrm{CsI}$ are currently used but have limitations, be it timing, stopping power, or toxicity (particularly in the case of $\mathrm{Cd}$ ).

A radiation hard scintillator operating at room temperature with a combination of high luminosity, short decay time, high stopping power and low afterglow is desired. There are more than 350 ternary tantalates

\footnotetext{
${ }^{*}$ Corresponding author.

Email address: smiadakd@msu.edu (David M. Smiadak)
}

(metal-Ta-O) compounds that can provide good stopping power for X-rays. Overall, only a few tantalates have been explored as scintillator material despite $\mathrm{Li}, \mathrm{Y}$, and $\mathrm{K}$ based tantalates proving to be efficient phosphors. $\mathrm{YTaO}_{4}, \mathrm{LaTaO}_{4}$ and $\mathrm{LuTaO}_{4}$ [3-6] are efficient luminescent materials at room temperature while the luminescence of other tantalates such as $\mathrm{SbTaO}_{4}$ or $\mathrm{BiTaO}_{4}$ is quenched at room temperature [7].

In this work, ternary tantalates, chosen for their ease of synthesis at ambient pressure, were produced as crystalline powders by solid-state synthesis and characterized for their scintillation properties using X-ray excitation. $\mathrm{Mg}_{4} \mathrm{Ta}_{2} \mathrm{O}_{9}$ was grown as a single crystal and its scintillation properties were confirmed.

\section{Experimental methods}

\subsection{Synthesis}

All samples were prepared by solid-state synthesis with starting reactants - tantalum oxide and other required metal oxides - of high purity ( $99.99 \%$ or better). The reactant powders were thoroughly ground together, transferred to an alumina reaction vessel, then reacted at appropriate temperatures and atmosphere, typically 
for 1 to 48 hours. Crystal growth of the compound $\mathrm{Mg}_{4} \mathrm{Ta}_{2} \mathrm{O}_{9}$ was demonstrated by the $\mu$-pull down technique. A larger single crystal was then obtained by the floating-zone technique in a mirror furnace to assess its scintillation properties.

\subsection{Single crystal growth}

A schematic diagram of the $\mu$-pull down apparatus (Cyberstar-Échirolles, France) is given in Figure 1 [8]. The $\mu$-pull down method provides directional solidification of a homogeneously melted single-phase compound that is seeded at the exit of a small capillary channel at the bottom of the crucible [9]. Melting point and chemical reactivity of the melt determine the choice of crucible material. Here the growth was performed using an iridium crucible and after-heater heated by radio frequency $(\mathrm{RF})$ through an inductive coil. Two layers of $\mathrm{Al}_{2} \mathrm{O}_{3}$ were used as heat insulators. The iridium crucible measured $16 \mathrm{~mm}$ in diameter and $30 \mathrm{~mm}$ in height with a nozzle diameter of $1 \mathrm{~mm}$ at the center of a flat $3 \mathrm{~mm}$ die. A hole was drilled through both the insulation and after-heater to view the molten zone and its meniscus with a CCD camera.

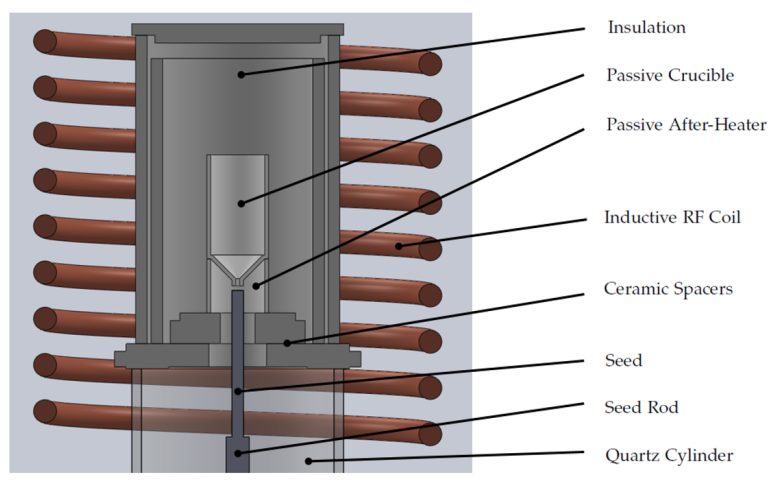

Figure 1: Schematic diagram of the $\mu$-PD apparatus [8].

The $\mu$-pull down chamber was evacuated and maintained under vacuum for 12 hours prior to the introduction of an inert atmosphere of argon that was circulated at a slightly positive pressure of $105 \mathrm{kPa}$ prior to initiating the heating profile. While not ideal for the growth of refractory oxides, an inert atmosphere is needed to prevent oxidation of the iridium crucible and after-heater. This pressure was maintained during growth with a constant gas flow rate of $20 \mathrm{ml} / \mathrm{min}$. Crystal growth was initiated at a rate varying from $0.05-0.26 \mathrm{~mm} / \mathrm{min}$.

\subsection{Characterization}

The crystallinity and structure identification of each sample was determined by powder X-ray diffraction.
An in-house diffraction setup comprised of a Bruker Nonius FR591 rotating anode X-ray generator equipped with a copper target and a $50 \mathrm{kV}$ and $60 \mathrm{~mA}$ electron beam was utilized. A more detailed description of this setup is supplied in [10].

All luminescence measurements were conducted at room temperature on samples consisting of micron-size crystalline particles contained in quartz cuvettes. X-ray excited emission spectra were measured for each sample using a second port of the Bruker Nonius X-ray generator and their spectral response was recorded by a SpectraPro-2150i spectrometer (Acton Research Corp., Acton, MA) coupled to a PIXIS:100B charge-coupled detector (CCD) (Princeton Instruments, Inc., Trenton, NJ).

Scintillation decay measurements were performed on each sample using a pulsed X-ray system that produces $80 \mathrm{ps}$ full width at half maximum (FWHM) pulses of Xrays having a mean energy of $18 \mathrm{keV}$. Fluorescent photons from each sample were detected by a microchannel phototube with 35 ps (FWHM) response. The luminescence decay times were determined by fitting data to a sum of exponential decay curves. The pulsed X-ray source, microchannel phototube, and fitting procedures are described in [11].

The reported estimated luminosities in Table 1 and 2 were determined using a $\mathrm{Bi}_{4} \mathrm{Ge}_{3} \mathrm{O}_{12}$ (BGO) sample as standard $(8,500 \mathrm{ph} / \mathrm{MeV})$ measured in the same conditions in particular, the same particle size [12].

Scintillation light yields were estimated by comparing the photopeak position of these crystals under gamma-ray excitation $\left({ }^{137} \mathrm{Cs}\right)$ to that of reference crystals as noted. All measurements were completed with a Hamamatsu R6231-100 photomultiplier tube (PMT) connected to an Ortec 113 preamplifier, an Ortec 672 spectroscopic amplifier and an Ortec EASY-MCA-8K multichannel analyzer. The PMT high voltage was fixed at $-650 \mathrm{~V}$. Samples were optically coupled onto the window of the PMT with Viscasil 600,000 (GE) and covered with layers of ultraviolet light reflecting tape (PTFE).

\section{Results}

\subsection{Screening of tantalate compounds for luminosity under $X$-ray excitation}

Tables 1 and 2 present the 63 tantalates that were synthesized and characterized for their scintillation properties. Sample composition and crystalline structure were confirmed by powder X-ray diffraction. All samples having an estimated luminosity of $150 \mathrm{ph} / \mathrm{MeV}$ and below are considered non-luminescent materials at room 
temperature. Compounds with luminosity values of less than $700 \mathrm{ph} / \mathrm{MeV}$ are listed in Table 1 while the more luminous compounds are listed in Table 2. Emission wavelengths are not listed for the majority of the compounds listed in Table 1. For those compounds listed, the detected luminescence was emitted in a very weak and broad band. Among those compounds, some may have luminescent potential at lower temperatures but it is beyond the scope of this work to report on quenching temperatures. No variable temperature measurements were performed. The emission wavelengths and decay times of the luminescence are listed for compounds in Table 2.

The compounds shaded in Table 1 have been studied previously for their luminescence but for which there were no data concerning the decay times of the X-ray excited luminescence. All samples showed a broad band emission with a FWHM of 150 to $200 \mathrm{~nm}$. The three most luminous compounds analyzed were: $\mathrm{Mg}_{2} \mathrm{Ta}_{2} \mathrm{O}_{9}$, $\mathrm{Li}_{3} \mathrm{TaO}_{4}$, and $\mathrm{Zn}_{3} \mathrm{Ta}_{2} \mathrm{O}_{8}$. The origin of luminescence in tantalate compounds is mainly attributed to the recombination of self-trapped excitons (STE), $\mathrm{Ta}^{4+}-\mathrm{O}^{-}$, on a tantalate group. Variation of the excitonic luminescence intensity among the compounds is interpreted in terms of crystal structure/self-trapping energy relationship [13-15]. The energy of the self-trapping decreases with increasing Ta-O-Ta angles, resulting in a stronger delocalization of STE's. We find a correlation between the luminosity and the Ta-O-Ta angles. Small Ta-O-Ta angles are correlated with higher luminosity, a relation previously noted by Blasse et al. [14, 15]. We also find that there are some exceptions to this rule, illustrated in Figure 2. As indicated in Eng et al. [16], factors such as the connectivity of the octahedra impact the band gap of the material, which influences luminosity.

Of the 63 synthesized tantalates, only 12 compounds had luminosity values exceeding 1,000 photons/MeV. Of these, most produced long decay times, $\mathrm{ScTaO}_{4}$ and $\mathrm{Zn}_{3} \mathrm{Ta}_{2} \mathrm{O}_{8}$ having the shortest, $(<1.5 \mu \mathrm{s})$. Two of the top three for luminosity, $\mathrm{Mg}_{2} \mathrm{Ta}_{2} \mathrm{O}_{9}$ and $\mathrm{Zn}_{3} \mathrm{Ta}_{2} \mathrm{O}_{8}$, have a density greater than $6 \mathrm{~g} / \mathrm{cm}^{3}$ which has the potential to increase the compounds stopping power. Further attention is given to these two compounds below.

\subsection{Impact of temperature synthesis on $\mathrm{Zn}_{3} \mathrm{Ta}_{2} \mathrm{O}_{8}$ stoi- chiometry}

$\mathrm{Zn}_{3} \mathrm{Ta}_{2} \mathrm{O}_{8}$ has been studied previously as a thin film material for low-voltage cathodoluminescence [17-19], where the mechanism of its blue luminescence was presented. Due to its relatively bright luminescence under X-ray at room temperature, and its high density, we

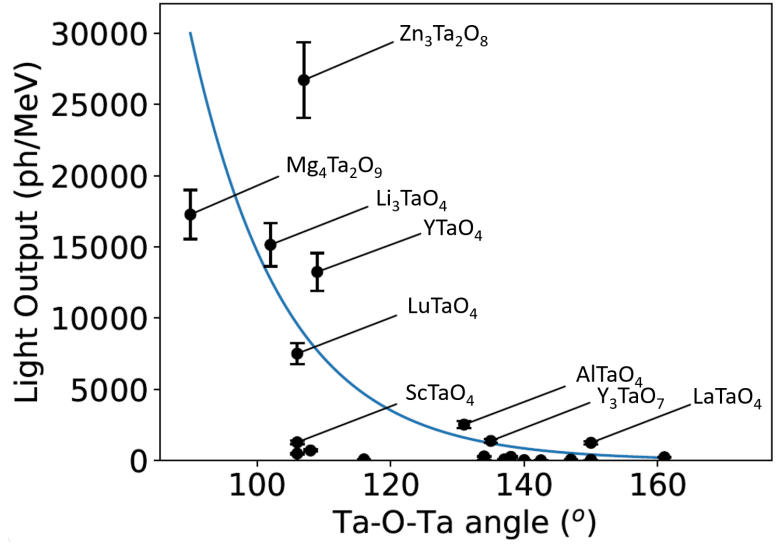

Figure 2: Relative luminosity of ternary tantalates vs the Ta-O-Ta angle in the structure. Four tantalates are outlined as not fitting the rule suggested in $[2,3]$.

present more details of data acquired under X-ray excitation for the crystalline powder (Table 1) and for its applications as a scintillator.

$\mathrm{Zn}_{3} \mathrm{Ta}_{2} \mathrm{O}_{8}$ was prepared by direct reaction of $\mathrm{ZnO}$ and $\mathrm{Ta}_{2} \mathrm{O}_{5}$ at temperature ranging from $1100^{\circ} \mathrm{C}$ to $1300^{\circ} \mathrm{C}$ for two to five hours. Due to the volatility of $\mathrm{ZnO}$ above $1200^{\circ} \mathrm{C}$, the solid-state reaction was also attempted with off-stoichiometry compositions utilizing excess $\mathrm{ZnO}$. Additionally, the reaction atmosphere was changed from air to argon and forming gas $\left(3 \% \mathrm{H}_{2} / 97 \%\right.$ $\mathrm{Ar})$. The pure phase was obtained after five hours at $1300^{\circ} \mathrm{C}$ in air from a stoichiometric composition of reactants.

The X-ray excited luminescence spectrum is shown in Figure 3. The broad emission peak is centered around $3.2 \mathrm{eV}$, typical of intrinsic emission in un-doped tantalate compounds. Its decay curve is shown in Figure 4, fitted by three exponential components yielded the results listed in Table 2 with a main component of approximately $1 \mu \mathrm{s}$. The $2 \mathrm{D}$ optical response, and emission spectrum as a function of excitation energy, is presented in Figure 5. The excitation and emission spectra are shown in Figure 6. The excitation spectrum was measured following the maximum of the emission at approximately $400 \mathrm{~nm}$ and the emission spectrum was recorded under $300 \mathrm{~nm}$ excitation. The emission is similar to the one observed under X-ray excitation.

\section{3. $\mathrm{Mg}_{4} \mathrm{Ta}_{2} \mathrm{O}_{9}$ single crystals grown by the $\mu-P D$ tech- nique}

The $\mathrm{MgO}-\mathrm{Ta}_{2} \mathrm{O}_{5}$ phase diagram is shown in Figure 7 [18]. Two stable phases exist in this system at room temperature: $\mathrm{Mg}_{4} \mathrm{Ta}_{2} \mathrm{O}_{9}$ and $\mathrm{MgTa}_{2} \mathrm{O}_{6}$. The dotted lines in- 
Table 1: Studied metal-tantalate-oxide with low luminosity values. Shaded compounds have been studied previously for their luminescence, however, no data concerning X-ray excited luminescence was collected.

\begin{tabular}{|c|c|c|c|c|c|}
\hline Compound & Synthesis & Crystal Structure & $\begin{array}{l}\text { Density } \\
\left(\mathrm{g} / \mathrm{cm}^{3}\right)\end{array}$ & $\begin{array}{l}\text { Emission } \\
(\mathrm{nm})\end{array}$ & $\begin{array}{c}\text { Luminosity } \\
(\mathrm{ph} / \mathrm{MeV})\end{array}$ \\
\hline $\mathrm{Ba}_{3} \mathrm{Ta}_{2} \mathrm{O}_{8}$ & $1,400^{\circ} \mathrm{C} / 10 \mathrm{~h} / \mathrm{Air}$ & Monoclinic & 7.29 & - & 64 \\
\hline $\mathrm{BaTa}_{2} \mathrm{O}_{6}$ & $1,400^{\circ} \mathrm{C} / 10 \mathrm{~h} / \mathrm{Air}$ & Hexagonal & 7.81 & - & 7 \\
\hline $\mathrm{BaTa}_{4} \mathrm{O}_{11}$ & $1,400^{\circ} \mathrm{C} / 10 \mathrm{~h} / \mathrm{Air}$ & Tetragonal & 8.07 & - & 7 \\
\hline $\mathrm{Bi}_{3} \mathrm{TaO}_{7}$ & $1,100^{\circ} \mathrm{C} / 10 \mathrm{~h} / \mathrm{Air}$ & Hexagonal & 9.32 & - & 30 \\
\hline $\mathrm{Bi}_{5} \mathrm{Ta}_{3} \mathrm{O}_{15}$ & $1,100^{\circ} \mathrm{C} / 10 \mathrm{~h} / \mathrm{Air}$ & Orthorhombic & 9.85 & - & 3 \\
\hline $\mathrm{BiTa}_{7} \mathrm{O}_{19}$ & $1,100^{\circ} \mathrm{C} / 10 \mathrm{~h} / \mathrm{Air}$ & Hexagonal & 8.8 & - & 33 \\
\hline $\mathrm{BiTaO}_{4}$ & $\begin{array}{l}800^{\circ} \mathrm{C} / 12 \mathrm{~h} \& \\
1,200^{\circ} \mathrm{C} / 13 \mathrm{~h} / \text { Air }\end{array}$ & Orthorhombic & 9.54 & - & 12 \\
\hline $\mathrm{Ca}_{2} \mathrm{Ta}_{2} \mathrm{O}_{7}$ & $1,500^{\circ} \mathrm{C} / 15 \mathrm{~h} / \mathrm{H}_{2}$ & Hexagonal & 6.43 & - & 38 \\
\hline $\mathrm{Ca}_{4} \mathrm{Ta}_{2} \mathrm{O}_{9}$ & $1,500^{\circ} \mathrm{C} / 15 \mathrm{~h} / \mathrm{H}_{2}$ & Orthorhombic & 5.56 & - & 60 \\
\hline $\mathrm{Ca}_{5} \mathrm{Ta}_{2} \mathrm{O}_{10}$ & $1,500^{\circ} \mathrm{C} / 15 \mathrm{~h} / \mathrm{H}_{2}$ & Cubic & 5.28 & 400,600 & 484 \\
\hline $\mathrm{CaTa}_{2} \mathrm{O}_{6}$ & $1,500^{\circ} \mathrm{C} / 15 \mathrm{~h} / \mathrm{H}_{2}$ & Orthorhombic & 7.19 & - & 257 \\
\hline $\mathrm{CaTa}_{4} \mathrm{O}_{11}$ & $1,600^{\circ} \mathrm{C} / 10 \mathrm{~h} / \mathrm{Air}$ & Hexagonal & 7.73 & - & 241 \\
\hline $\mathrm{Cd}_{2} \mathrm{Ta}_{2} \mathrm{O}_{7}$ & $1,050^{\circ} \mathrm{C} / 10 \mathrm{~h} / \mathrm{Air}$ & Cubic & 8.41 & 640 & 270 \\
\hline $\mathrm{CeTaO}_{4}$ & $1,600^{\circ} \mathrm{C} / 10 \mathrm{~h} / \mathrm{Air}$ & Monoclinic & 8.41 & - & 50 \\
\hline $\mathrm{CeTa}_{3} \mathrm{O}_{9}$ & $1,500^{\circ} \mathrm{C} / 10 \mathrm{~h} / \mathrm{Air}$ & Monoclinic & 8.88 & - & 7 \\
\hline $\mathrm{CsTa}_{5} \mathrm{O}_{14}$ & $1,100^{\circ} \mathrm{C} / 10 \mathrm{~h} / \mathrm{Air}$ & Orthorhombic & 6.79 & - & 45 \\
\hline $\mathrm{GaTaO}_{4}$ & $1,400^{\circ} \mathrm{C} / 10 \mathrm{~h} / \mathrm{Air}$ & Orthorhombic & 7.46 & - & 115 \\
\hline $\mathrm{GdTa}_{3} \mathrm{O}_{9}$ & $1,500^{\circ} \mathrm{C} / 10 \mathrm{~h} / \mathrm{N}_{2}$ & & - & - & 194 \\
\hline $\mathrm{GdTa}_{7} \mathrm{O}_{19}$ & $1,100^{\circ} \mathrm{C} / 10 \mathrm{~h} / \mathrm{Air}$ & Tetragonal & 8.49 & - & 36 \\
\hline $\mathrm{GdTaO}_{4}$ & $1,600^{\circ} \mathrm{C} / 10 \mathrm{~h} / \mathrm{Air}$ & Monoclinic & 8.83 & 520 & 698 \\
\hline $\mathrm{Hf}_{6} \mathrm{Ta}_{2} \mathrm{O}_{17}$ & $1,300^{\circ} \mathrm{C} / 10 \mathrm{~h} / \mathrm{Air}$ & Orthorhombic & 8.9 & - & 38 \\
\hline $\mathrm{InTaO}_{4}$ & $1,400^{\circ} \mathrm{C} / 10 \mathrm{~h} / \mathrm{Air}$ & Monoclinic & 7.2 & - & 15 \\
\hline $\mathrm{KTaO}_{3}$ & $1,000^{\circ} \mathrm{C} / 2 \mathrm{~h} / \mathrm{Air}$ & Cubic & 7.02 & - & 277 \\
\hline $\mathrm{La}_{3} \mathrm{TaO}_{7}$ & $1,500^{\circ} \mathrm{C} / 10 \mathrm{~h} / \mathrm{Air}$ & Orthorhombic & 7.13 & 475 & 38 \\
\hline $\mathrm{LaTa}_{3} \mathrm{O}_{9}$ & $1,500^{\circ} \mathrm{C} / 10 \mathrm{~h} / \mathrm{Air}$ & Orthorhombic & 8.7 & - & 69 \\
\hline $\mathrm{LaTa}_{5} \mathrm{O}_{14}$ & $1,600^{\circ} \mathrm{C} / 10 \mathrm{~h} / \mathrm{Air}$ & Orthorhombic & 7.32 & - & 31 \\
\hline $\mathrm{LaTa}_{7} \mathrm{O}_{19}$ & $1,500^{\circ} \mathrm{C} / 10 \mathrm{~h} / \mathrm{Air}$ & Hexagonal & 8.45 & - & 258 \\
\hline $\mathrm{Lu}_{3} \mathrm{TaO}_{7}$ & $1,500^{\circ} \mathrm{C} / 10 \mathrm{~h} / \mathrm{Air}$ & Cubic & 9.76 & 350 & 178 \\
\hline $\mathrm{MgTa}_{2} \mathrm{O}_{6}$ & $1,300^{\circ} \mathrm{C} / 10 \mathrm{~h} / \mathrm{Air}$ & Tetragonal & 7.81 & 380 & 398 \\
\hline $\mathrm{MoTa}_{2} \mathrm{O}_{11}$ & $600^{\circ} \mathrm{C} / 10 \mathrm{~h} / \mathrm{Air}$ & Rhombohedral & 6.15 & - & 20 \\
\hline $\mathrm{Na}_{2} \mathrm{Ta}_{2} \mathrm{O}_{6}$ & $750^{\circ} \mathrm{C} / 15 \mathrm{~h} / \mathrm{Air}$ & Cubic & 5.88 & - & 16 \\
\hline $\mathrm{Na}_{2} \mathrm{Ta}_{4} \mathrm{O}_{11}$ & $750^{\circ} \mathrm{C} / 15 \mathrm{~h} / \mathrm{Air}$ & Rhombohedral & 7.74 & - & 35 \\
\hline $\mathrm{Na}_{2} \mathrm{Ta}_{8} \mathrm{O}_{21}$ & $750^{\circ} \mathrm{C} / 15 \mathrm{~h} / \mathrm{Air}$ & Orthorhombic & 7.56 & - & 22 \\
\hline $\mathrm{Na}_{5} \mathrm{Ta}_{11} \mathrm{O}_{30}$ & $750^{\circ} \mathrm{C} / 15 \mathrm{~h} / \mathrm{Air}$ & Rhombohedral & 7.33 & - & 32 \\
\hline $\mathrm{Na}_{5} \mathrm{TaO}_{5}$ & $750^{\circ} \mathrm{C} / 15 \mathrm{~h} / \mathrm{Air}$ & Monoclinic & 4.02 & 380 & 698 \\
\hline $\mathrm{NaTaO}_{3}$ & $1,300^{\circ} \mathrm{C} / 10 \mathrm{~h} / \mathrm{Air}$ & Monoclinic & 7.06 & - & 10 \\
\hline $\mathrm{Pb}_{2} \mathrm{Ta}_{2} \mathrm{O}_{7}$ & $900^{\circ} \mathrm{C} / 10 \mathrm{~h} / \mathrm{Air}$ & Hexagonal & 9.34 & - & 14 \\
\hline $\mathrm{Pb}_{3} \mathrm{Ta}_{4} \mathrm{O}_{13}$ & $900^{\circ} \mathrm{C} / 10 \mathrm{~h} / \mathrm{Air}$ & Cubic & 9.54 & - & 10 \\
\hline $\mathrm{Pb}_{5} \mathrm{Ta}_{4} \mathrm{O}_{15}$ & $900^{\circ} \mathrm{C} / 10 \mathrm{~h} / \mathrm{Air}$ & Hexagonal & 9.45 & - & 28 \\
\hline $\mathrm{Pb}_{22} \mathrm{Ta}_{18} \mathrm{O}_{67}$ & $900^{\circ} \mathrm{C} / 10 \mathrm{~h} / \mathrm{Air}$ & Rhombohedral & 9.44 & - & 50 \\
\hline $\mathrm{PbTa}_{2} \mathrm{O}_{6}$ & $900^{\circ} \mathrm{C} / 10 \mathrm{~h} / \mathrm{Air}$ & Tetragonal & 9.02 & - & 18 \\
\hline $\mathrm{Sr}_{4} \mathrm{Ta}_{4} \mathrm{O}_{15}$ & $1,500^{\circ} \mathrm{C} / 15 \mathrm{~h} / \mathrm{Air}$ & Hexagonal & 7.29 & 400 & 328 \\
\hline $\mathrm{SrTa}_{2} \mathrm{O}_{6}$ & $1,600^{\circ} \mathrm{C} / 15 \mathrm{~h} / \mathrm{H}_{2}$ & Orthorhombic & 7.67 & - & 50 \\
\hline $\mathrm{SrTa}_{4} \mathrm{O}_{11}$ & $1,650^{\circ} \mathrm{C} / 10 \mathrm{~h} / \mathrm{H}_{2}$ & Hexagonal & 7.06 & - & 84 \\
\hline $\mathrm{W}_{2} \mathrm{Ta}_{30} \mathrm{O}_{81}$ & $1,400^{\circ} \mathrm{C} / 10 \mathrm{~h} / \mathrm{Air}$ & Orthorhombic & 8.32 & - & 105 \\
\hline $\mathrm{W}_{4} \mathrm{Ta}_{22} \mathrm{O}_{67}$ & $1,400^{\circ} \mathrm{C} / 10 \mathrm{~h} / \mathrm{Air}$ & Orthorhombic & 8.6 & - & 10 \\
\hline $\mathrm{W}_{9} \mathrm{Ta}_{8} \mathrm{O}_{47}$ & $1,400^{\circ} \mathrm{C} / 10 \mathrm{~h} / \mathrm{Air}$ & Orthorhombic & 7.55 & - & 5 \\
\hline $\mathrm{Yb}_{3} \mathrm{TaO}_{7}$ & $1,500^{\circ} \mathrm{C} / 10 \mathrm{~h} / \mathrm{Air}$ & Cubic & 9.62 & - & 13 \\
\hline $\mathrm{YbTaO}_{4}$ & $1,600^{\circ} \mathrm{C} / 10 \mathrm{~h} / \mathrm{Air}$ & Monoclinic & 9.67 & - & 26 \\
\hline $\mathrm{YTa}_{7} \mathrm{O}_{19}$ & $1,500^{\circ} \mathrm{C} / 10 \mathrm{~h} / \mathrm{Air}$ & Hexagonal & 8.32 & 360 & 600 \\
\hline $\mathrm{ZnTa}_{2} \mathrm{O}_{6}$ & $1300^{\circ} \mathrm{C} / 10 \mathrm{~h} / \mathrm{Air}$ & Orthorhombic & 8.53 & - & 288 \\
\hline
\end{tabular}




\begin{tabular}{|c|c|c|c|c|c|c|}
\hline Compound & Synthesis & Crystal Structure & $\begin{array}{l}\text { Density } \\
\left(\mathrm{g} / \mathrm{cm}^{3}\right)\end{array}$ & $\begin{array}{c}\text { Emission } \\
(\mathrm{nm})\end{array}$ & $\begin{array}{l}\text { Luminosity } \\
(\mathrm{ph} / \mathrm{MeV})\end{array}$ & $\begin{array}{l}\text { Major decays, } \\
\% \text { Light Yield }\end{array}$ \\
\hline $\mathrm{AlTaO}_{4}$ & $1,500^{\circ} \mathrm{C} / 10 \mathrm{~h} / \mathrm{Air}$ & Tetragonal & 8.28 & 350 & 1,900 & $\begin{array}{c}0.3 \mu \mathrm{s}(5 \%), \\
2.5 \mu \mathrm{s}(16 \%), \\
4.1 \mu \mathrm{s}(18 \%)\end{array}$ \\
\hline $\mathrm{CdTa}_{2} \mathrm{O}_{6}$ & $1,050^{\circ} \mathrm{C} / 10 \mathrm{~h} / \mathrm{Air}$ & Orthorhombic & 8.51 & 400 & 2,600 & $\begin{array}{c}6.2 \mu \mathrm{s}(37 \%), \\
>30 \mu \mathrm{s}(60 \%)\end{array}$ \\
\hline $\mathrm{LaTaO}_{4}$ & $1,600^{\circ} \mathrm{C} / 10 \mathrm{~h} / \mathrm{Air}$ & Monoclinic & 7.79 & 515 & 1,400 & $\begin{array}{c}0.3 \mu \mathrm{s}(17 \%) \\
0.9 \mu \mathrm{s}(20 \%) \\
9 \mu \mathrm{s}(61 \%)\end{array}$ \\
\hline $\mathrm{Li}_{3} \mathrm{TaO}_{4}$ & $1,400^{\circ} \mathrm{C} / 10 \mathrm{~h} / \mathrm{Air}$ & Rhombohedral & 5.87 & 400 & 17,400 & $\begin{array}{l}6.1 \mu \mathrm{s}(65 \%) \\
>30 \mu \mathrm{s}(30 \%)\end{array}$ \\
\hline $\mathrm{LuTaO}_{4}$ & $\begin{array}{l}800^{\circ} \mathrm{C} / 5 \mathrm{~h} / \mathrm{Air} \& \\
1,500^{\circ} \mathrm{C} / 10 \mathrm{~h} / \mathrm{Air}\end{array}$ & Monoclinic & 9.75 & 330 & 9,500 & $\begin{array}{l}1.4 \mu \mathrm{s}(26 \%) \\
0.7 \mu \mathrm{s}(16 \%), \\
3.1 \mu \mathrm{s}(35 \%)\end{array}$ \\
\hline $\mathrm{Mg}_{4} \mathrm{Ta}_{2} \mathrm{O}_{9}$ & $1,300^{\circ} \mathrm{C} / 10 \mathrm{~h} / \mathrm{Air}$ & Hexagonal & 6.17 & 350 & 19,900 & $\begin{array}{l}3.9 \mu \mathrm{s}(71 \%), \\
>17 \mu \mathrm{s}(27 \%)\end{array}$ \\
\hline $\mathrm{ScTaO}_{4}$ & $1,400^{\circ} \mathrm{C} / 10 \mathrm{~h} / \mathrm{Air}$ & Monoclinic & 6.9 & 360 & 1,500 & $\begin{array}{c}0.2 \mathrm{~ms}(14 \%), \\
0.5 \mathrm{~ms}(18 \%), \\
1.4 \mathrm{~ms}(59 \%)\end{array}$ \\
\hline $\mathrm{Y}_{10} \mathrm{Ta}_{4} \mathrm{O}_{25}$ & $1,500^{\circ} \mathrm{C} / 10 \mathrm{~h} / \mathrm{Air}$ & Orthorhombic & 7.13 & 360 & 3,900 & $\begin{array}{l}0.3 \mu \mathrm{s}(8 \%) \\
2.1 \mu \mathrm{s}(87 \%)\end{array}$ \\
\hline $\mathrm{Y}_{3} \mathrm{TaO}_{7}$ & $1,500^{\circ} \mathrm{C} / 10 \mathrm{~h} / \mathrm{Air}$ & Orthorhombic & 6.4 & 360 & 1,600 & $\begin{array}{l}1.4 \mu \mathrm{s}(60 \%) \\
2.4 \mu \mathrm{s}(10 \%)\end{array}$ \\
\hline $\mathrm{YTa}_{3} \mathrm{O}_{9}$ & $1,600^{\circ} \mathrm{C} / 10 \mathrm{~h} / \mathrm{Air}$ & & & 365 & 2,500 & $\begin{array}{l}1.7 \mu \mathrm{s}(75 \%) \\
5.5 \mu \mathrm{s}(15 \%)\end{array}$ \\
\hline $\mathrm{YTaO}_{4}$ & $1,600^{\circ} \mathrm{C} / 10 \mathrm{~h} / \mathrm{Air}$ & Monoclinic & 7.57 & 420 & 15,200 & $\begin{array}{l}0.7 \mu \mathrm{s}(11 \%) \\
3.8 \mu \mathrm{s}(62 \%)\end{array}$ \\
\hline $\mathrm{Zn}_{3} \mathrm{Ta}_{2} \mathrm{O}_{8}$ & $1,300^{\circ} \mathrm{C} / 10 \mathrm{~h} / \mathrm{Air}$ & Monoclinic & 7.14 & 400 & 26,000 & $\begin{array}{c}0.2 \mu \mathrm{s}(20 \%) \\
1.1 \mu \mathrm{s}(80 \%)\end{array}$ \\
\hline
\end{tabular}




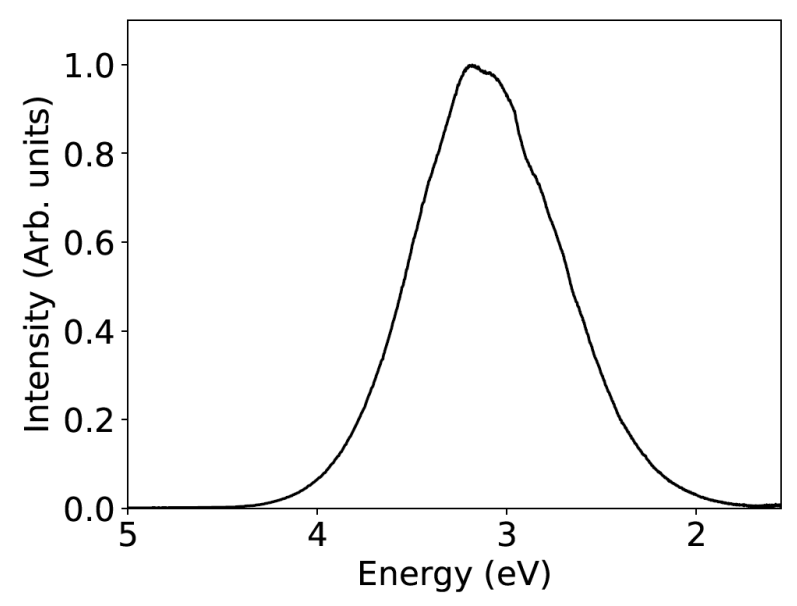

Figure 3: X-ray excited luminescence emission spectra of $\mathrm{Zn}_{3} \mathrm{Ta}_{2} \mathrm{O}_{8}$.

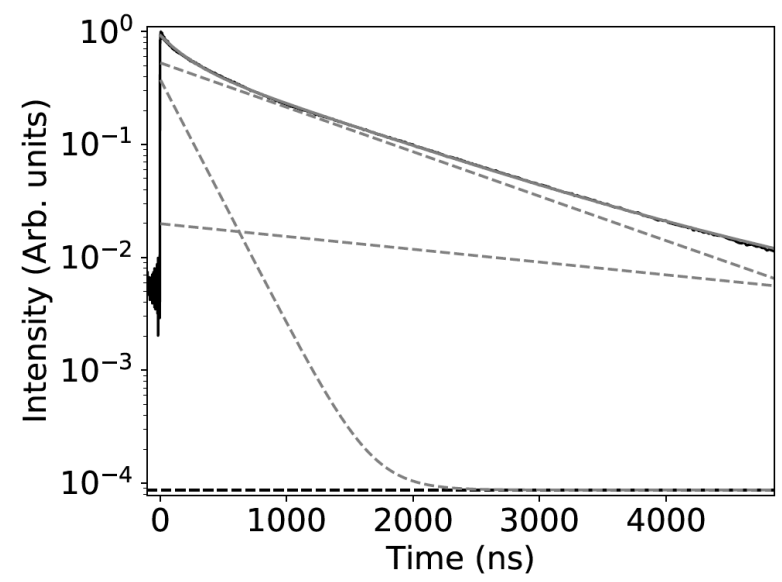

Figure 4: Decay curves of the X-ray excited luminescence acquired at room temperature.

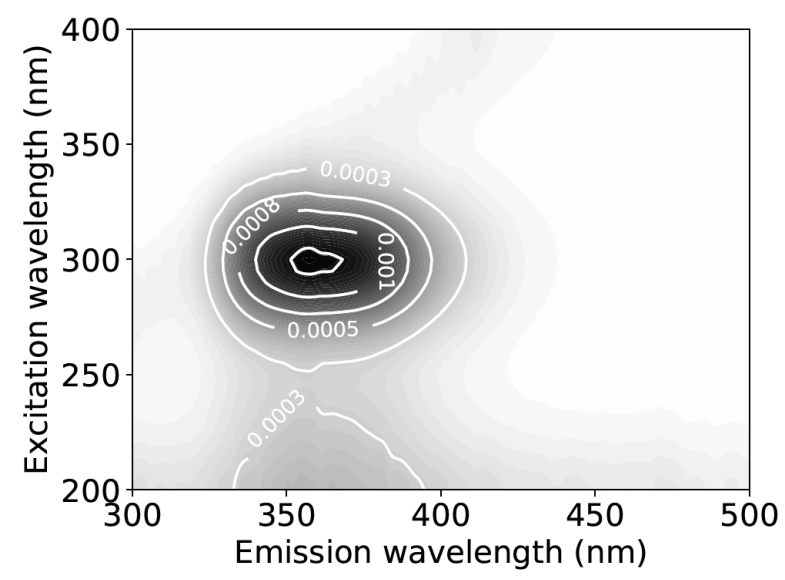

Figure 5: $\mathrm{Zn}_{3} \mathrm{Ta}_{2} \mathrm{O}_{8} 2 \mathrm{D}$ optical response.

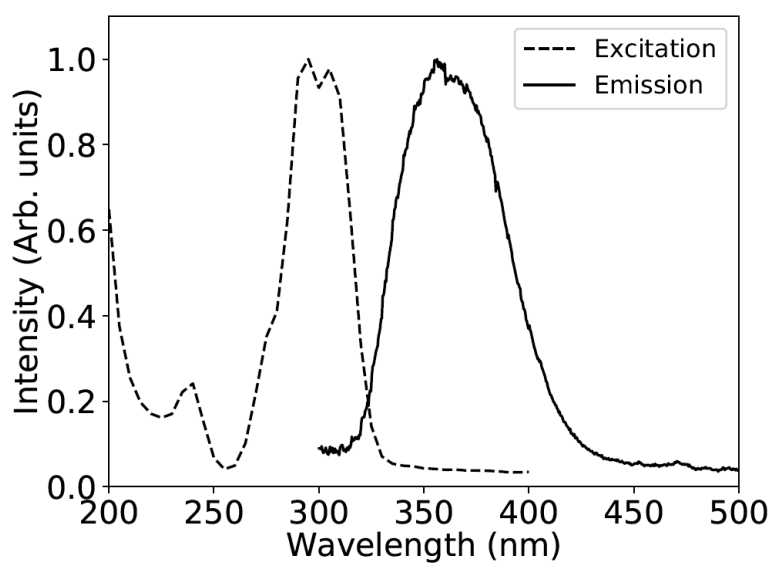

Figure 6: $\mathrm{Zn}_{3} \mathrm{Ta}_{2} \mathrm{O}_{8}$ optical excitation and emission.

dicate some uncertainties, specifically around the melting point of $\mathrm{Mg}_{4} \mathrm{Ta}_{2} \mathrm{O}_{9}$. Additionally, it is unclear if $\mathrm{Mg}_{4} \mathrm{Ta}_{2} \mathrm{O}_{9}$ melts congruently.

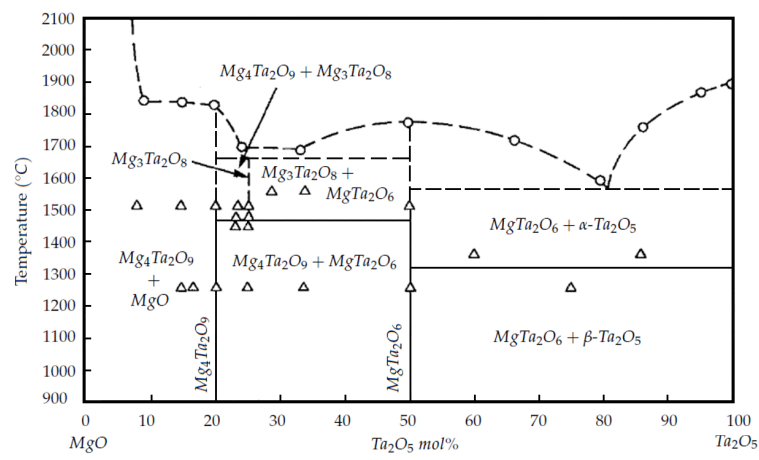

Figure 7: Phase diagram of the $\mathrm{MgO}-\mathrm{Ta}_{2} \mathrm{O}_{5}$ system reproduced from reference [18].

While a crystal was grown previously by the optical floating zone technique [19] further optimization was required to produce an optically clear crystal. The $\mu$ PD technique (Figure 1) was selected for growth as it is well-suited for growing crystals rapidly with small diameters (1 to $4 \mathrm{~mm}$ ) and lengths on the cm scale [9] without the use of a crucible.

Lacking a $\mathrm{Mg}_{4} \mathrm{Ta}_{2} \mathrm{O}_{9}$ single crystal seed to initiate growth, the first crystals were seeded with a $\mathrm{Y}_{3} \mathrm{Al}_{5} \mathrm{O}_{12}$ (YAG) crystal, then self-seeded thereafter. Once the growth parameters were optimized for power input and growth rate, a long single crystal of the material could be produced. A number of growths are shown in Figure 8. Confirmation of the phase was performed by X-ray powder diffraction (Figure 9).

Structure determination was carried out by single 


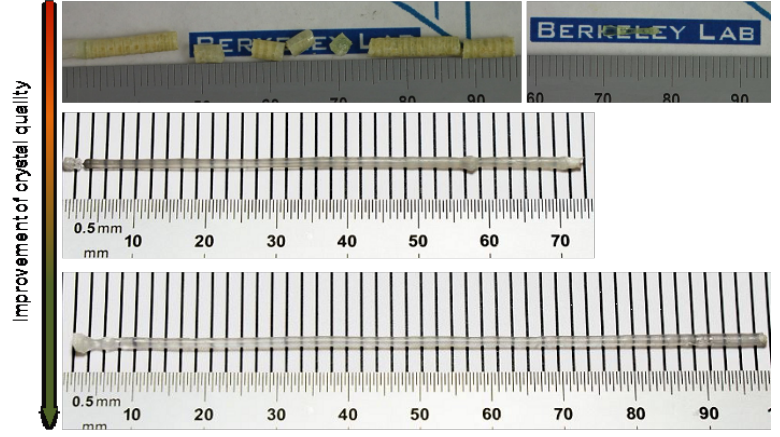

Figure 8: Optimization of the growth process for $\mathrm{Mg}_{4} \mathrm{Ta}_{2} \mathrm{O}_{9}$ crystals. The crystal shown at the bottom is clear and uniform with a diameter of about $1.5 \mathrm{~mm}$ and a length of about $9.5 \mathrm{~cm}$.

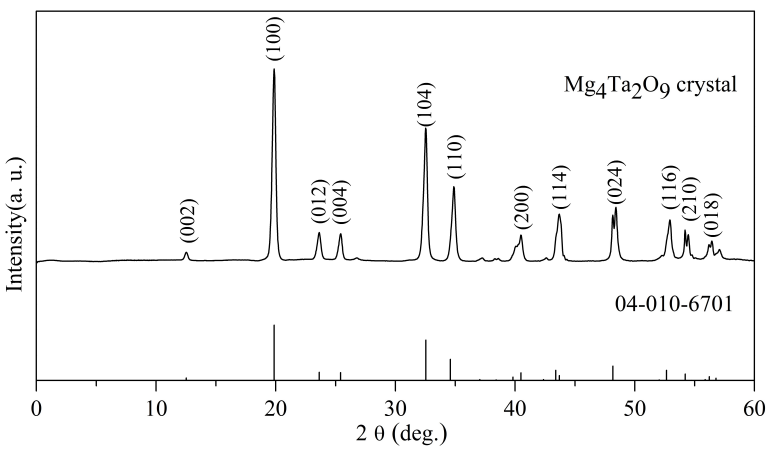

Figure 9: Powder diffraction patterns of $\mathrm{Mg}_{4} \mathrm{Ta}_{2} \mathrm{O}_{9}$ : grown crystal (top), and PDF reference (bottom).

crystal X-ray diffraction on a Bruker Kappa Apex II diffractometer using Mo-K $\alpha$ radiation. Samples for measurement were cut from the middle portion of each grown crystal. Single crystal pieces were then mounted on glass fibers and transferred to the diffractometer. The Apex SMART program was used to determine the unit cell parameters (data collection of $5 \mathrm{~s} /$ frame, $0.3^{\circ} /$ frame for a sphere of diffraction data). The raw frame data was processed using the SAINT program [20]. Empirical absorption correction was applied based on multiscan. Data were collected at 150 and $325 \mathrm{~K}$ using an Oxford nitrogen gas cryostream system. Subsequent calculations were carried out using the SHELXTL program [21].

$\mathrm{Mg}_{4} \mathrm{Ta}_{2} \mathrm{O}_{9}$ crystallizes in the trigonal group $\mathrm{P} \overline{3} \mathrm{c} 1$ (165), with unit cell parameters a $=5.1625(10) \AA, c=$ 14.062(4) A with a calculated density of $6.17125 \mathrm{~g} / \mathrm{cm}^{3}$. A schematic of the structure is shown in Figure 10.

The crystal obtained by the $\mu$-PD technique was subsequently used as a seed in the floating-zone growth (Figure 11) to produce crystals approximately 3-5 mm

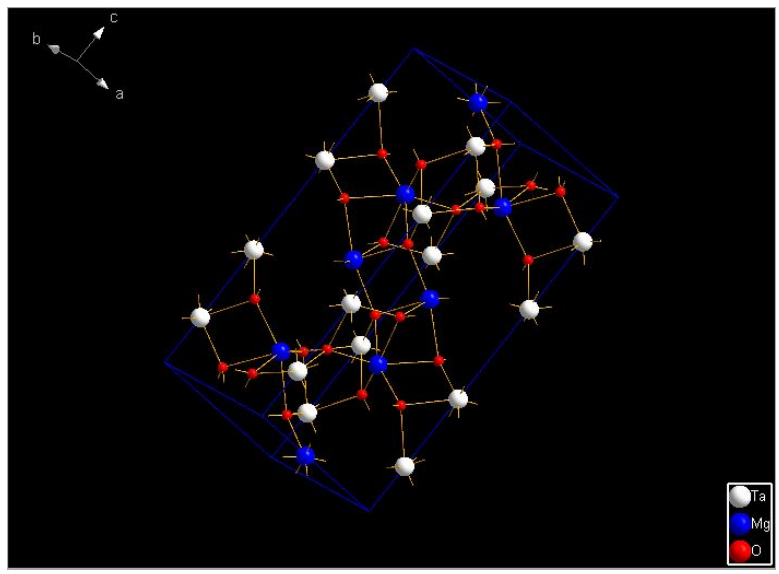

Figure 10: Single crystal structure determination of $\mathrm{Mg}_{4} \mathrm{Ta}_{2} \mathrm{O}_{9}$ with structure parameters indicated in text.

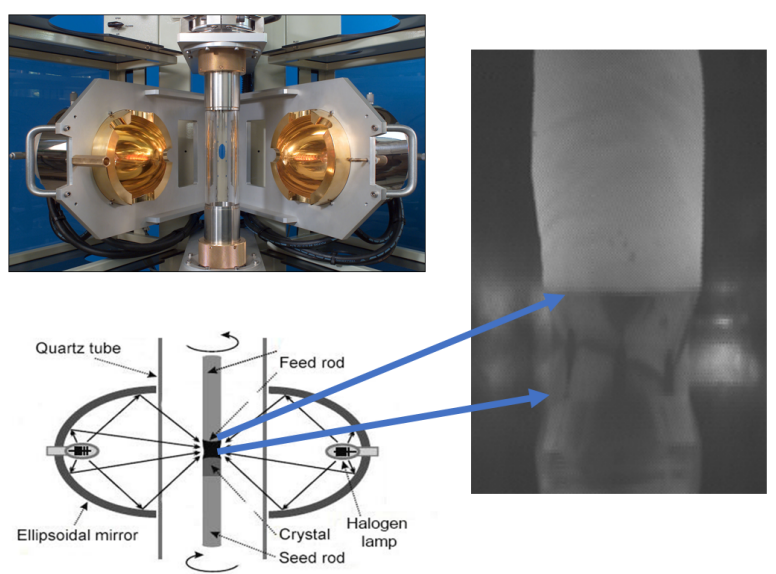

Figure 11: Single crystal growth in the mirror furnace.

in diameter. The feed rod was prepared from sintered powder of stoichiometric composition.

Four crystals were grown with improving quality from the first to the fourth, as shown in Figure 12, labeled A-D. All crystals suffered from the same issues of bubble formation and cracking previously reported for the $\mathrm{MgTa}_{2} \mathrm{O}_{6}$ [19]. Despite these defects, optically clear pieces were recovered for luminosity and energy resolution measurements.

\subsubsection{Optical and scintillation properties}

Inhomogeneities in the crystals resulted in variations of the X-ray excited luminescence spectrum as shown in Figure 13. In the screening experiments, it was determined that the emission peak of pure $\mathrm{Mg}_{4} \mathrm{Ta}_{2} \mathrm{O}_{9}$ was centered at approximately $350 \mathrm{~nm}$. The same emission peak was seen for crystal B with a FWHM of approx- 


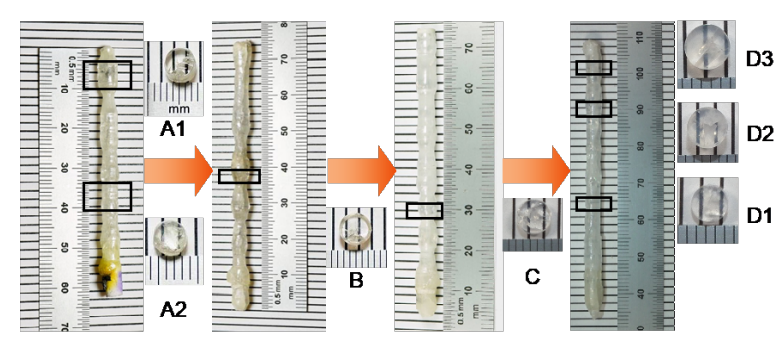

Figure 12: Four crystals of $\mathrm{Mg}_{4} \mathrm{Ta}_{2} \mathrm{O}_{9}$ grown in the mirror furnace. The arrows indicate the chronology of growth and marked improvements. The third and fourth crystals were grown with an excess of $1 \%$ and $0.5 \%$ mole $\mathrm{MgO}$ respectively, in the ceramic feed rod and the overall best crystal (B) was grown at $2 \mathrm{~mm} / \mathrm{hr}$. The growth rates were $0.3,2,1$ and $2 \mathrm{~mm} / \mathrm{hr}$ for crystals A, B, C and D respectively. Slices were cut in the clear portion of the crystals as shown then polished for scintillation measurements.

imately $75 \mathrm{~nm}$. The other crystals exhibited broader emission bands shifted to lower energies, an additional broad band extending from 650 to $900 \mathrm{~nm}$ was observed in the emission spectrum of crystal D.

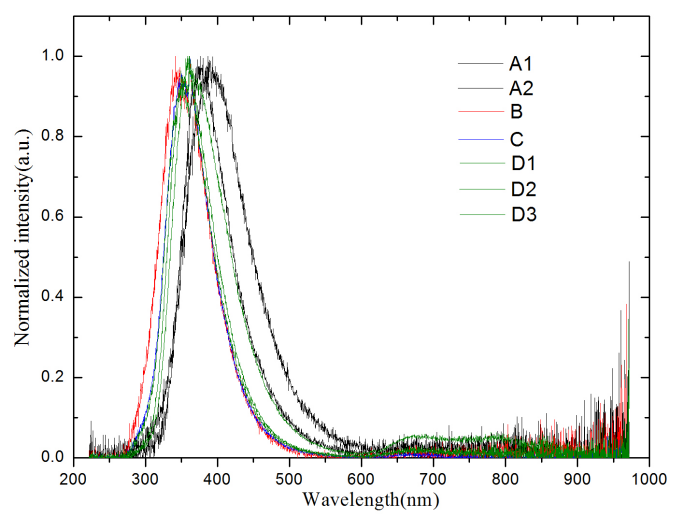

Figure 13: Emission spectra of single crystal samples of $\mathrm{Mg}_{4} \mathrm{Ta}_{2} \mathrm{O}_{9}$ measured under optical excitation at room temperature.

A small rod with dimensions $1 \times 3 \times 2 \mathrm{~mm}$, was cut from crystal B and used for pulse height measurements under gamma excitation with results shown in Figure 13. $\mathrm{CdWO}_{4}, \mathrm{CaWO}_{4}$ and BGO were measured in identical conditions for comparison. Values of light output calculated with correction for wavelengths of emission are given in Table 2. It was determined that the $\mathrm{Mg}_{4} \mathrm{Ta}_{2} \mathrm{O}_{9}$ single crystal had a luminosity comparable to that of $\mathrm{CdWO}_{4}$ and $\mathrm{CaWO}_{4}$ and may possess a better energy resolution.

While there are variations in the results due to inhomogeneities in the crystals, selected data was indicative

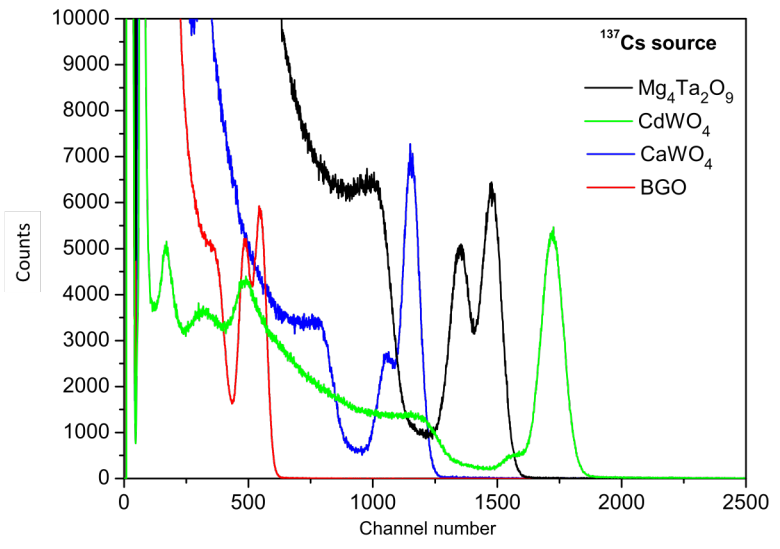

Figure 14: Comparison of pulse height spectra of $\mathrm{Mg}_{4} \mathrm{Ta}_{2} \mathrm{O}_{9}$ with those of $\mathrm{CdWO}_{4}, \mathrm{CaWO}_{4}$ and $\mathrm{Bi}_{4} \mathrm{Ge}_{3} \mathrm{O}_{12}$ measured under gamma ray excitation $\left({ }^{137} \mathrm{Cs}\right)$. Measurements at room temperature.

\begin{tabular}{cccc}
\hline Crystal & Size $(\mathrm{mm})$ & $\mathrm{LO}(\mathrm{ph} / \mathrm{MeV})$ & $\mathrm{ER}, \% @ 662 \mathrm{keV}$ \\
\hline $\mathrm{Bi}_{4} \mathrm{Ge}_{3} \mathrm{O}_{12}$ & $1 \times 2 \times 2$ & $8,000-10,000$ & 6.4 \\
$\mathrm{CaWO}_{4}$ & $3 \times 10 \times 10$ & 15,000 & 6.75 \\
$\mathrm{CdWO}_{4}$ & $5 \times 5 \times 5$ & $12,000-15,000$ & 8.3 \\
$\mathrm{Mg}_{4} \mathrm{Ta}_{2} \mathrm{O}_{9}$ & $1 \times 3 \times 2$ & $13,000 \pm 2,000$ & 6.2 \\
\hline
\end{tabular}

Table 3: Measured values of luminosity and energy resolution for a small $\mathrm{Mg}_{4} \mathrm{Ta}_{2} \mathrm{O}_{9}$ single crystal and comparison with $\mathrm{CdWO}_{4}$, $\mathrm{CaWO}_{4}$ and $\mathrm{Bi}_{4} \mathrm{Ge}_{3} \mathrm{O}_{12}$.

of what can be achieved in luminosity and energy resolution. With improved crystal quality, the performance should also improve. Further work on the growth of these crystals will be published separately.

\section{Conclusions}

There is a large variation in the scintillation properties (luminosity, decay time, emission wavelength) of the analyzed tantalate compounds. Of the 63 tantalates screened as crystalline powders, $\mathrm{Zn}_{3} \mathrm{Ta}_{2} \mathrm{O}_{8}$ was found to have the best combination of density and luminescence at room temperature. $\mathrm{Zn}_{3} \mathrm{Ta}_{2} \mathrm{O}_{8}$ is a dense material $\left(7.147 \mathrm{~g} / \mathrm{cm}^{3}\right)$ and pure crystalline $\mathrm{Zn}_{3} \mathrm{Ta}_{2} \mathrm{O}_{8}$ powders have an estimated luminosity of $26,000 \mathrm{ph} / \mathrm{MeV}$ with a main decay time of approximately 600 ns. However melt growth of this compound at low pressure is not possible due to the high pressure of $\mathrm{ZnO}$ above $1200^{\circ} \mathrm{C}$. High pressure growth was not attempted.

Small $\mathrm{Mg}_{4} \mathrm{Ta}_{2} \mathrm{O}_{9}$ single crystals were grown exhibiting a luminosity comparable to that of $\mathrm{CdWO}_{4}$ and $\mathrm{CaWO}_{4}$. Further work on the growth optimization of 
$\mathrm{Mg}_{4} \mathrm{Ta}_{2} \mathrm{O}_{9}$ has the potential to produce crystals with improved energy resolution.

\section{Acknowledgments}

This document was prepared as an account of work sponsored by the United States Government. While this document is believed to contain correct information, neither the United States Government nor any agency thereof, nor The Regents of the University of California, nor any of their employees, makes any warranty, express or implied, or assumes any legal responsibility for the accuracy, completeness, or usefulness of any information, apparatus, product, or process disclosed, or represents that its use would not infringe privately owned rights. Reference herein to any specific commercial product, process, or service by its trade name, trademark, manufacturer, or otherwise, does not necessarily constitute or imply its endorsement, recommendation, or favoring by the United States Government or any agency thereof, or The Regents of the University of California. The views and opinions of authors expressed herein do not necessarily state or reflect those of the United States Government or any agency thereof or The Regents of the University of California. This work was supported by the US Department of Homeland Security, Domestic Nuclear Detection Office, Nuclear Science and Security Consortium Fellowship and carried out at Lawrence Berkeley National Laboratory under Contract NO. AC02-05CH11231.

\section{References}

[1] A.A. Annenkov, M.V. Korzhik, and P. Lecoq. Lead tungstate scintillation material. Nuclear Inst. and Methods in Physics Research, A, 490(1):30-50, 2002.

[2] P. Lecoq, A. Annenkov, A. Gektin, M. Korzhik, and C. Pedrini. Inorganic Scintillators for Detector Systems: Physical Principles and Crystal Engineering. Springer, Cham, 2006.

[3] G. Blasse and A. Bril. Luminescence phenomena in compounds with fergusonite structure. Journal of Luminescence, 3(2):109$131,1970$.

[4] G. Blasse, G.J. Dirksen, L.H. Brixner, and M.K. Crawford. Luminescence of materials based on $\mathrm{LuTaO}_{4}$. Journal of Alloys and Compounds, 209(1):1-6, 1994.

[5] M.J. Weber, S. Derenzo, C. Dujardin, and W.W. Moses. Dense $\mathrm{Ce} 3^{+}$-activated scintillator materials. In Proceedings of the International Conference on Inorganic Scintillators and Their Applications, pages 325-328, 011995.

[6] L.I. Kazakova, A.B. Dubovsky, G.V. Semenkovich, and O.A. Ivanova. Luminescence of $\mathrm{YTaO}_{4}$ single crystals. Radiation Measurements, 24(4):359-360, 1995. Proceedings of the International Symposium on Luminescent Detectors and Transformers of Ionizing Radiation.

[7] M. Wiegel, W. Middel, and G. Blasse. Influence of $\mathrm{ns}^{2}$ ions on the luminescence of niobates and tantalates. J. Mater. Chem., 5:981-983, 1995.
[8] D.M. Smiadak. Scintillator candidate compounds. Master's thesis, Michigan State University, 2015.

[9] T. Fukuda and V.I. Chani. Shaped Crystals: Growth by Micro-Pulling-Down Technique, volume 8. Springer-Verlag, Berlin/Heidelberg, 1 edition, 2007.

[10] S.E. Derenzo, M.S. Boswell, E. Bourret-Courchesne, R. Boutchko, T.F. Budinger, A. Canning, S.M. Hanrahan, M. Janecek, Q. Peng, Y. Porter-Chapman, J.D. Powell, C.A. Ramsey, S.E. Taylor, L.W. Wang, M.J. Weber, and D.S. Wilson. Design and implementation of a facility for discovering new scintillator materials. IEEE Transactions on Nuclear Science, 55(3):1458-1463, 2008.

[11] S.E. Derenzo, M.J. Weber, W.W. Moses, and C. Dujardin. Measurements of the intrinsic rise times of common inorganic scintillators. IEEE Transactions on Nuclear Science, 47(3):860$864,2000$.

[12] Martin Janecek, Ramesh Borade, Edith Bourret-Courchesne, and Stephen E. Derenzo. An investigation of x-ray luminosity versus crystalline powder granularity. Nuclear Instruments and Methods in Physics Research, A, 659(1):252-257, 2011.

[13] G. Blasse and L.H. Brixner. Luminescence of perovskite-like niobates and tantalates. Materials Research Bulletin, 24(3):363$366,1989$.

[14] G. Blasse and L. De Haart. The nature of the luminescence of niobates $\mathrm{MNbO}_{3}(\mathrm{M}=\mathrm{Li}, \mathrm{Na}, \mathrm{K})$. Materials Chemistry and Physics, 14(5):481-484, 1986.

[15] L.G.J. De Haart, A.J. De Vries, and G. Blasse. On the photoluminescence of semiconducting titanates applied in photoelectrochemical cells. Journal of Solid State Chemistry, 59(3):291300, 1985.

[16] H. W. Eng, P.W. Barnes, B.M. Auer, and P.M. Woodward. Investigations of the electronic structure of $\mathrm{d} 0$ transition metal oxides belonging to the perovskite family. Journal of Solid State Chemistry, 175(1):94-109, 2003.

[17] P.D. Rack, M.D. Potter, A. Woodard, and S. Kurinec. Negative ion resputtering in $\mathrm{Ta}_{2} \mathrm{Zn}_{3} \mathrm{O}_{8}$ thin films. Journal of Vacuum Science $\mathcal{E}$ Technology A: Vacuum, Surfaces, and Films, 17(5):2805-2810, 1999.

[18] Y. Baskin and D.C. Schell. Phase studies in the binary system $\mathrm{MgO}-\mathrm{Ta}_{2} \mathrm{O}_{5}$. Journal of the American Ceramic Society, 46(4):174-177, 1963.

[19] M. Higuchi, K. Ando, J. Takahashi, and K. Kodaira. Growth of mgta $\mathrm{O}_{6}$ single crystals by floating zone method and their optical properties. Journal of the Ceramic Society of Japan, 101(1169):118-120, 1993.

[20] Bruker AXS Inc., Madison, Wisconsin. APEX2 and SAINT, 2006.

[21] G.M. Sheldrick. A short history of shelx. Acta Crystallographica Section A, 64(1):112-122, 2008. 


\section{Cranfield University}

\section{CERES https://dspace.lib.cranfield.ac.uk}

2018-05-25

\section{Scintillation of tantalate compounds}

\section{Bourret, Edith D.}

\section{Elsevier}

Bourret ED, Smiadak DM, Borade RB, et al., Scintillation of tantalate compounds, Journal of Luminescence, Volume 202, October 2018, pp. 332-338

http://dx.doi.org/10.1016/j.jlumin.2018.05.044

Downloaded from Cranfield Library Services E-Repository 